\title{
Apuntes para un mapa de la poesía hispanoamericana en Brasil, \\ 1990-2020
}

Notes for a map of the HispanicAmerican poetry in Brazil, 1990-2020

Chayenne Orru Mubarack

Idalia Morejón Arnaiz

Recebido em: 11 de novembro de 2020

Aceito em: 30 de novembro de 2020
Doutoranda em Letras/Espanhol pela Universidade de São Paulo. Contato: chayenne.mubarack@ usp.br

Brasil

Escritora e professora de literatura hispano-americana da Faculdade de Filosofia, Letras e Ciências Humanas da Universidade de São Paulo. Contato: idamorejon@usp.br Brasil 


\section{PALABRAS CLAVE:}

Poesía hispanoamericana en Brasil; Revistas de poesía en Brasil; Traducción de poesía hispanoamericana en Brasil.

\section{KEYWORDS:}

Hispanicamerican Poetry in Brazil; Literature magazines in Brazil; Translation of hispanicamerican poetry in Brazil.
Resumen: Este artículo presenta un breve relato del proyecto de investigación La traducción y publicación de poesía hispanoamericana en Brasil, 1990-2020 desarrollado por estudiantes de grado de la disciplina de Literatura Hispanoamericana de la Facultad de Filosofía, Letras y Ciencias Humanas de la Universidad de São Paulo. Identificamos un corpus de publicaciones relevantes y presentamos algunas conclusiones sobre la configuración de un posible mapa poético hispanoamericano en Brasil durante las últimas cuatro décadas, a partir del análisis estadístico de los datos obtenidos.

Abstract: This article presents a brief report of the research project "The translation and the publication of Hispanicamerican poetry in Brazil, 1990-2020" developed by undergraduates in the Hispanicamerican Literature assignment of the Philosophy, Letters and Human Sciences Faculty at the University of São Paulo. We have selected a corpus composed of relevant masazines and some conclusions will be presented about the configuration of a possible Hispanicamerican poetic map in Brazil during the past four decades, from the statistical analysis of the obtained data. 
EL PROYECTO Y LAS REVISTAS

Este artículo presenta un relato del proyecto de investigación " $\mathrm{La}$ traducción y publicación de poesía hispanoamericana en Brasil, 1990-2010” desarrollado por estudiantes de grado del Departamento de Letras Modernas, vinculados a la disciplina de Literatura Hispanoamericana, entre 2014 y 2019. El mismo se propuso mapear la poesía hispanoamericana traducida y publicada en Brasil con vistas a contribuir a la introducción de los estudiantes en la investigación con revistas literarias, buscando resultados específicos a través de la preparación de trabajos académicos. El proyecto estuvo centrado, en un primer momento, en la localización y fichaje de datos en las revistas de poesía de mayor circulación en Brasil en el mismo período. Partimos de los archivos existentes en la hemeroteca de la biblioteca Florestan Fernandes, de la Facultad de Filosofía, Letras y Ciencias Humanas de la Universidad de São Paulo, de la Biblioteca Brasiliana Guita e José Mindlin, y los centros culturales paulistas Casa das Rosas, Centro Cultural São Paulo, Itaú Cultural y la Biblitoeca Mário de Andrade, de cuyos acervos aprovechamos las colecciones de revistas, en muchos casos incompletas, que nos han permitido trazar un mapa poético de contornos aún imprecisos: Agulha (1999), Azougue (2003), Babel (2000), Duas águas (1997), Inimigo rumor (1997-2008), Mallarmargens (2012), Musa Rara (2010), Outra travessia (2003), Poesia sempre (1993), Polichinello (2004), Sibila (2001), Transluminura (2013) y Zunái (2003). Además de consultar publicaciones impresas, la búsqueda se concentró en algunas de las revistas más visibles no solo por utilizar plataformas digitales, sino también por su duración: Sibila, Zunái y Agulha. 
A lo largo de los cinco años en que el proyecto contó con el apoyo del Programa Unificado de Bolsas (PUB) de la Universidad de São Paulo, un total de nueve estudiantes ${ }^{1}$ fueron entrenados en los principios teóricos y metodológicos para el estudio de revistas literarias, por tanto, el procesamiento de los datos que aquí presentaremos es el resultado de un esfuerzo colectivo, docente y discente, que tuvo como ejes de reflexión cuestiones teóricas y críticas vinculadas a la poesía de Hispanoamérica y su traducción al portugués de Brasil, con vistas a comprender las relaciones establecidas entre poetas y traductores de dicho país, así como de otros países de América latina, a través de las revistas de poesía. Además de llevar a cabo este mapeo de la poesía, los datos colectados nos permitieron analizar la recepción de dicha poesía en Brasil, en específico, los modos de circulación de las poéticas postvanguardistas hispanoamericanas en su intersección con las poéticas concretistas y postconcretistas brasileñas, lo cual nos ayudó a pensar la poesía como dispositivo de transculturación. Esto fue posible gracias a la implementación de una serie de objetivos específicos, organizados alrededor de la potencial capacidad de los estudiantes para el trabajo de investigación: profundizar el conocimiento de la lengua española, fundamentalmente por medio de la lectura supervisada de poesía; realizar búsquedas bibliográficas, tanto en bases de datos informatizadas como en bibliotecas y hemerotecas; fomentar la habilidad de los estudiantes para identificar aspectos esenciales

1 Estudiantes que participaron en las diferentes etapas del proyecto, entre 2014 y 2019: Larissa Pavoni Rodrigues, Camila Barbosa de Oliveira, Caroline Costa Pereira, Cristiane Gomes, Chayenne Mubarack, Nicholas Magno Evans, Kelly Cavalheiro, Mikhael Oliveira Simóes y Wesley Rocha. 
del trabajo de investigación (definir fundamentos, estrategias de análisis, objetivos y metas en pesquisas de carácter filológico y artístico, orientadas desde la óptica de la literatura y la crítica literaria hispanoamericana); familiarizar a los estudiantes con los recursos necesarios para realizar un aprendizaje autónomo de la poesía hispanoamericana.

Como resultados específicos, el proyecto produjo dos trabajos de graduación individual (TGI) sobre las revistas Inimigo Rumor (Larissa Pavoni) ${ }^{2}$ y Sibilia (Camila Barbosa de Oliveira) $)^{3}$. Chayenne Orru Mubarack (2016) produjo un artículo (inédito) sobre la revista Transluminura (a partir del segundo número pasó a llamarse Circuladô) de la Casa das Rosas, en el cual analiza la configuración epigonal de la revista en su primer número, marcado por el magisterio de Haroldo de Campos, con el objetivo de mostrar su contacto con la diversidad de la poesía brasileña e hispanoamericana contemporánea, y de manera más autónoma, con relación al concretismo. Durante la etapa final del proyecto, Chayenne Orru Mubarack investigó la recepción del poeta cubano José Kozer en dichas revistas de poesía, así como sus vínculos con la estética neobarroca brasileńa. También la estudiante Caroline Costa Pereira (2016) publicó una entrevista a Floriano Martins, director de la revista Agulha. Otra cuestión relevante que fue observada durante la segunda fase de recopilación de informaciones sobre la revista

2 Rodrigues, Larissa Pavoni. "Poesia argentina na Inimigo Rumor: tradução e recepção". Trabalho de Graduação Individual. Faculdade de Filosofia, Letras e Ciências Humanas da USP, 2015.

3 Oliveira, Camila Barbosa de. "A poesia da neovanguarda cubana na revista Sibila". Trabalho de Graduação Individual. Faculdade de Filosofia, Letras e Ciências Humanas da USP, 2015. 
Sibila se refiere a los cambios de configuración ocurridos en la misma, a partir de su transformación en página web, pensando sobre todo en un modo de analizar teórica y poéticamente dicho cambio.

\section{Plataformas}

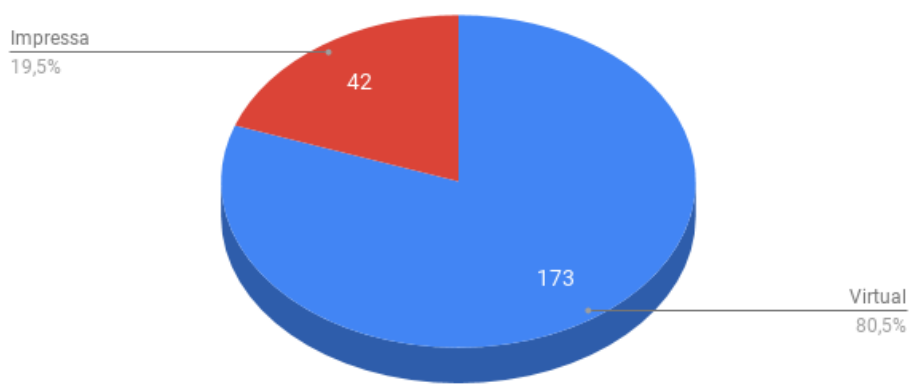

Figura 1 - Plataformas

Entre 2018 y 2019, Wesley Rocha organizó todas las informaciones recogidas tanto en las revistas como en los catálogos editoriales disponibles, y elaboró una serie de infográficos que formalizan los resultados del proyecto, exclusivamente a partir del corpus de publicaciones a que tuvimos acceso. A esto agregamos, durante el último ańo del proyecto, un levantamiento de las antologías de poesía hispanoamericana publicadas por editoriales brasileñas, tanto aquellas con una visibilidad expresiva en el mercado editorial, como las independientes. $\mathrm{Al}$ incorporar a las editoriales ampliamos el período de análisis en diez años, o sea, de 1990 a 2020, ya que en la última década han surgido nuevas revistas en línea que debemos revisar, y la presencia de editoriales independientes con 
proyectos traductológicos sólidos se ha hecho más notable 4 . Por otro lado, esto nos permitirá evaluar con mayor profundidad los mecanismos de recepción de la poesía de lengua española publicada en Brasil.

El cumplimiento de los objetivos antes mencionados fue lo que nos permitió identificar un corpus de publicaciones relevantes para la difusión de la poesía hispanoamericana en Brasil y llegar a algunas conclusiones sobre la configuración de un posible mapa poético hispanoamericano en algunas regiones de Brasil (norte, sudeste y sur) durante las últimas tres décadas, a partir del análisis estadístico de los datos obtenidos.

\section{Revista}

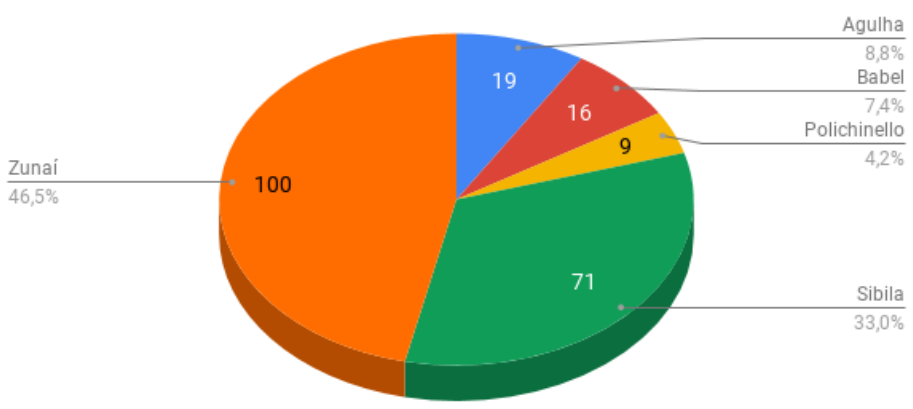

Figura 2 - Revista

Verificamos, en primera instancia, el lugar secundario que ocupa la poesía hispanoamericana en territorio brasileño, donde la traducción de poesía de

4 Entre los proyectos de traducción recientes más notables se encuentra el catálogo de Edições Jabuticaba. Disponible en: https://www.edicoesjabuticaba.com.br/pd-55a672-nocaute-6-poetascuba-hoje-org-jose-ramon-sanchez.html?ct=\&p=1\&s=1. Acceso el 20 ene. 21. 
lengua inglesa predomina en el mercado editorial, y cuya mayor circulación se ubica en la ciudad de Sáo Paulo (Milton, 2004). En segundo lugar, observamos que los criterios para la selección de la poesía hispanoamericana publicada en las revistas brasileńas apuntan a un interés por la traducción de poemas de autores vivos y a movimientos específicos como el surrealismo (Agulha), el neobarroco y, en menor medida, la poesía conceptual (Zunái, Sibila). Respecto a la presencia del surrealismo, Floriano Martins, director de Agulha hace la siguiente distinción:

esta não é uma publicação formalmente ligada ao movimento surrealista. A presença destacada do Surrealismo em nossas páginas resulta de sua importância inquestionável, de sua influência perene, no que tange à criação artística em praticamente todo o mundo. No entanto, lidamos com uma pauta aberta, dando ao leitor condiçóes de criar seu próprio espaço de leitura, de preferência, de amizade.

\section{$[\ldots]$}

tais projetos se confundem em um só, e não poderia ser de outro modo. Porém não se limitam ao Surrealismo. Não são coordenadas atreladas ao movimento e sim uma rota que permite averiguar existência e valores reais do Surrealismo entre nós. O que se passa é que foi um movimento extremamente relevante para as artes e o pensamento no Século XX, mantendo até hoje uma visceralidade renovada, de que é prova a recente ediçâo de uma exposição internacional do surrealismo na Costa Rica, da qual participei com obras e com um dos ensaios do catálogo. Há também um aspecto histórico, o fato de que, sobretudo no Brasil, o Surrealismo foi muito castigado pelo preconceito e a certa intenção estratégica da parte de alguns protagonistas de nossa cultura. Mas tanto a revista quanto meus livros de ensaios se abrem para outros panoramas, outras investigaçóes, e mesmo em relação ao Surrealismo não há perda de sentido crítico, ou seja, náo há um acento ortodoxo em momento algum. De todos modos, é um dado fundamental este cuidado que tenho com trazer à tona o mapa 
real de atuação do Surrealismo em nosso continente, de que são exemplos um livro de estudos que recentemente foi publicado no México e uma versão bem mais ampliada do mesmo que agora nos preparamos para publicar pela ARC Ediçóes.

Constatamos además que en el período que va de la década de 1990 a la primera de los años 2000 tiene lugar una transición en el modo de concebir la traducción de poesía. La revista Inimigo Rumor, por ejemplo, comienza con traducciones autorales para posteriormente incorporar traducciones colectivas, sin embargo, esa modalidad no se extiende a lo restante del corpus de las revistas analizadas. En ese sentido, entre los números especiales de Inimigo Rumor, el número 7 de 1999 presentó cinco nuevos poetas argentinos, indicados por el poeta y traductor Aníbal Cristobo ${ }^{5}$ quien, tras su ingreso al comité editorial de la revista, tradujo e incluyó poemas de autores hispanoamericanos poco divulgados en Brasil.

Por otra parte, encontramos un énfasis en la traducción de poetas vinculados al neobarroco, entre los cuales el cubano José Kozer es quien mayor visibilidad ha alcanzado hasta el presente. Al interrogarnos sobre la presencia notable de la poesía neobarroca verificamos que esto ocurre

5 En la actualidad Aníbal Cristobo reside en Barcelona y allí dirige la editorial Kriller71, cuyo catálogo se ha especializado en la publicación de poesía contemporánea, en la que la poesía brasileña y su propio trabajo como traductor tienen enorme relevancia. Además de Un útero es del tamaño de un puño, de Angélica Freitas (2016), y El libro de las semejanzas, de Ana Martins Marques (2019), traducidos por Paula Abramo, Cristobo firma las traducciones de: Paulo Leminski, Yo iba a ser Homero (2018); André Sant'anna, Amor (2016); Carlito Azevedo, Monodrama (2015); Marília Garcia, Error geográfico (2015); Luca Argel, Me olvidé de fijar el grafito (2015); Ricardo Domeneck, Ciclo del amante sustituible (2014); Marcos Siscar, La mitad del arte (2014); Manoel Ricardo Lima, Cuando todos los accidentes suceden (2014); Arnaldo Antunes, Instanto (2013). Disponible en: http://kriller71ediciones.com/libros/coleccion-kriller71/. Acceso 20 ene. 21. 
a través del contacto de poetas brasileños, que son también traductores (Josely Vianna Baptista, Claudio Daniel, Régis Bonvicino, Floriano Peixoto) con revistas o editoriales hispanoamericanas. De igual modo, la biblioteca personal de Haroldo de Campos ${ }^{6}$, ha contribuido a la difusión de la poesía neobarroca hispanoamericana en Brasil, ya que fue a través de los vínculos personales de este autor con Octavio Paz y Severo Sarduy que toda una red de poetas-traductores fue organizándose en revistas, editoriales y cuadernos culturales, tanto en la región sudeste como en el sur de Brasil. No menos importante fue la presencia del argentino Néstor Perlongher en São Paulo en los años 1980/1990, cuando organiza la antología Caribe Transplatino (1991), un marco en esta reunión de poetas alrededor del neobarroco/neobarroso.

El trabajo con las fichas comprueba la importancia de José Kozer en el panorama de las revistas literarias brasileñas de 1990 a 2020. Un ejemplo de ello es la revista Musa Rara, de la cual también consideramos los artículos que mencionan al poeta cubano. Esto pone en evidencia la manera como Kozer es visto como contrapunto para el análisis de otros autores, como aparece en artículos de Claudio Daniel y Floriano Martins. Ambos, al referirse a Nelson Ascher y Sergio Campos, respectivamente. Por otro lado, en artículos que tienen como objeto a las revistas literarias brasileñas, y es el caso de "Brazilian Poetry Journals of the 21st Century" y "Revistas definem

6 Disponible en: http://www.casadasrosas.org.br/centro-de-referencia-haroldo-de-campos/acervo. Acceso 20 ene. 21. 
o panorama literário", el nombre de Kozer siempre es citado debido a las colaboraciones realizadas.

La mayor parte de los poemas de su autoría publicados en las revistas analizadas se mantienen en la lengua original. En las escasas ocasiones en que aparecen traducidos, como en la revista Zunái, encontramos los textos en español y sus versiones al portugués. Uno de los motivos para esto podría ser el hecho de que en la poesía neobarroca el lenguaje es de extrema relevancia, así, al dejar ambas versiones, el lector tiene la posibilidad de cotejar los poemas. Respecto a los ensayos de Kozer, no existe tampoco homogeneidad en las traducciones. Mientras que la revista Musa Rara optó por publicar su ensayo "Volver a Thoreau" en español solamente, Zunái publicó, en traducción de Vima Teixeira, "Neobarroco: um convergente padrão na poesia latino-americana" ${ }^{\text {. En }}$ dicho ensayo, Kozer presenta dos líneas en la poesía latinoamericana actual. La primera sería una línea fina, cuya poesía es lineal, de expresión familiar y coloquial, y en ella convergen la poesía estadounidense y la latinoamericana. La segunda línea, gruesa y espesa, estaría compuesta por la poesía internacional, con diversidad más fuerte y abarcadora. Aquí, según Kozer, se alinean la poesía americana del siglo XX, la fuente del barroco de los siglos de oro, los poetas ingleses metafísicos, Mallarmé y Ezra Pound. En el escenario latinoamericano, la línea fina, durante

7 Para dar continuidad al estudio sobre la presencia de Kozer en las revistas brasileñas de poesía sugerimos consultar, además, las revistas Oroboro, Cacto y Et Cetera, a las cuales no tuvimos acceso. 
la primera mitad del siglo XX, estaba compuesta por Pablo Neruda, Octavio Paz, Nicanor Parra, Heberto Padilla y Eliseo Diego. Aparecería también, en este escenario específico, una línea intermediaria, en la cual estarían César Vallejo y Oliverio Girondo. Por fin, la línea espesa, en la que el propio Kozer localiza su escritura, incluiría a José Lezama Lima y Haroldo de Campos. Ambos estiran el lenguaje y se apropian de las libertades del barroco para formular el neobarroco en su propia lógica. Tal como Paulo Leminski, Roberto Echavarren, Eduardo Milán, David Huerta, Víctor Sosa, Claudio Daniel, Josely Vianna Baptista, Glauco Mattoso, Tamara Kamenszain, Arturo Carrera y Reynaldo Jiménez, autores que presentan la poesía en sí, sin contexto, y con la necesidad de que el lector realice una inmersión en el texto, cuya lectura será demorada. Así, concluye Kozer, el poeta neobarroco es un instrumento de la poesía, y no la poesía. Dentro de la llamada línea espesa Kozer identifica, como en el boxeo, tres modelos básicos: uno pesado, en el cual incluye a Wilson Bueno y Reynaldo Jiménez; uno mediano, con Roberto Echavarren y Néstor Perlongher como representantes, y por último uno leve, que abriga a Raúl Zurita y David Huerta, entre otros.

"Neobarroco: um convergente padrão na poesia latino-americana" es muestra del modo cómo Kozer vincula la poesía brasileńa y la hispanoamericana a partir del entramado neobarroco, acentuando con esto la importancia de esta poética para pensar el tema de la traducción de la poesía hispanoamericana en Brasil, así como la propia relevancia de las producciones poéticas y ensayísticas de este autor. 
Constatamos, por tanto, la presencia de Kozer a partir de distintas vertientes. La primera sería en tanto poeta, cuya producción fue publicada ostensivamente en revistas literarias brasileñas, tanto en español como en portugués. Por otro lado, podemos situarlo como ensayista, si consideramos la publicación de sus textos sobre el neobarroco. A partir de otra perspectiva, también resulta interesante observar la manera como en algunos textos críticos, aun cuando el poeta cubano no sea el objeto central, sí figura como contrapunto. Esto se observa, por ejemplo, en el artículo "Revistas definem o panorama literário", escrito por Claudio Daniel para la revista Musa Rara. En el mismo, José Kozer es señalado como exponente del neobarroco. Otro ejemplo en que Kozer aun no siendo objeto de análisis es citado como parámetro ocurre en el texto "Viagem por um mar interior", de Floriano Martins. El texto comenta el libro Mar anterior, de Sergio Campos, quien se interesaba por la poesía de Kozer, y también del peruano Javier Sologuren. Floriano señala dos semejanzas entre la escritura de Campos y la de Kozer: la prodigiosidad y vertiente abismal de una mitología personal, y la afirmación de una realidad otra, enriquecida por la memoria y por el curso incesante de sus descubrimientos.

No deja de resultar curioso, a su vez, que siendo el cubano el poeta más publicado en las revistas de poesía brasileña, le corresponda a la poesía argentina el mayor número de colaboraciones registradas, lo cual podemos comprobar en los siguientes gráficos: 


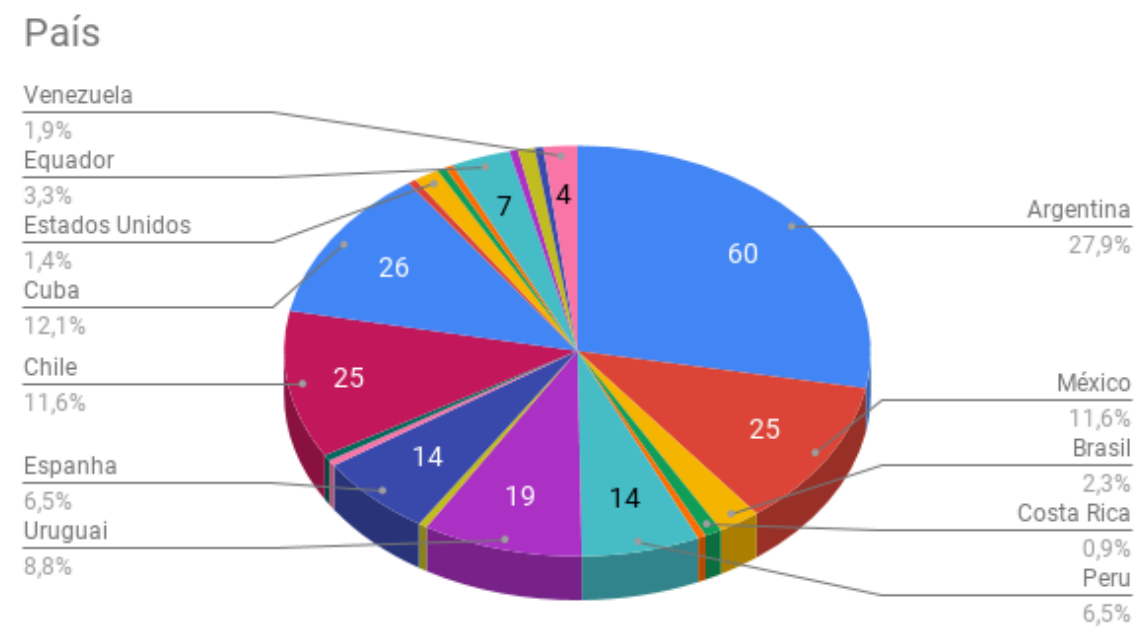

Figura SEQ Figura \* ARABIC 3 - País

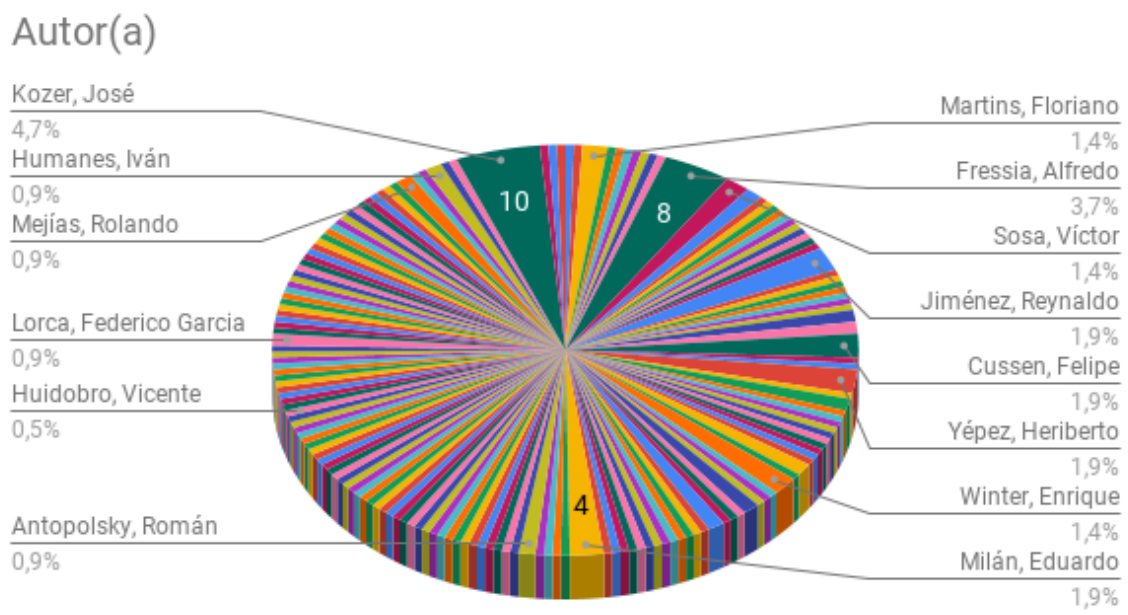

Figura SEO Figura \* ARABIC 4 - Autor(a) 
El primer gráfico representa a los países de origen de los poetas con mayor número de textos publicados en revistas literarias brasileñas. El segundo gráfico, a su vez, muestra a los autores más publicados. Esa contradicción -José Kozer con mayor visibilidad y mayor número de colaboradores argentinos- confirma que existe un interés, en el escenario de las revistas brasileñas, por el neobarroco, lo que justificaría la visibilidad del cubano, como uno de los mayores exponentes de esta estética.

\section{LAS EDITORIALES}

Verificamos que las editoriales de mayor productividad y circulación en el mercado del libro brasileño son las que menos han contribuido a la visibilidad de la poesía hispanoamericana, siendo pues las editoriales independientes las que han sostenido una política editorial en que la poesía gana protagonismo. Una de las más destacadas en ese sentido es la paulista Lumme Editor ${ }^{8}$, fundada en 2000 por el poeta Francisco dos Santos.

8 Disponible en: https://lummeeditor.com/. Acceso 20 ene. 21. 


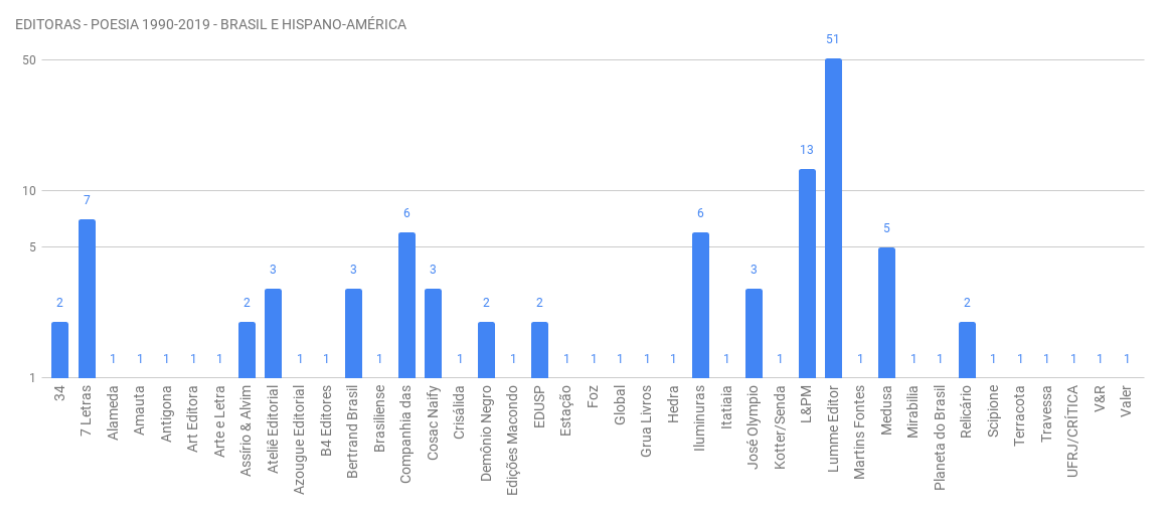

Figura 5 - Editoras - Poesia - Brasil e Hispanoamérica

$\mathrm{Al}$ analizar los países de origen de los autores publicados por Lumme Editor, la poesía cubana se destaca nuevamente. Sin embargo, a diferencia de las revistas, en la escena editorial independiente, Cuba es el país con más autores publicados, conforme podemos observar en el siguiente gráfico:

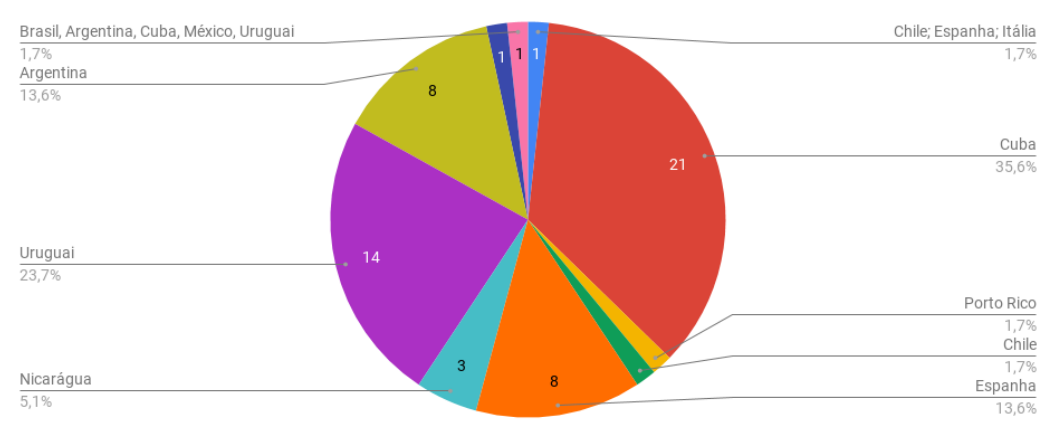

Figura 6 - Lumme Editor - Países 
Desde otra perspectiva, ahora pensando en los autores con más publicaciones en Lumme Editor, nuevamente es José Kozer el autor que más se destaca, en este caso, junto al uruguayo Víctor Sosa:

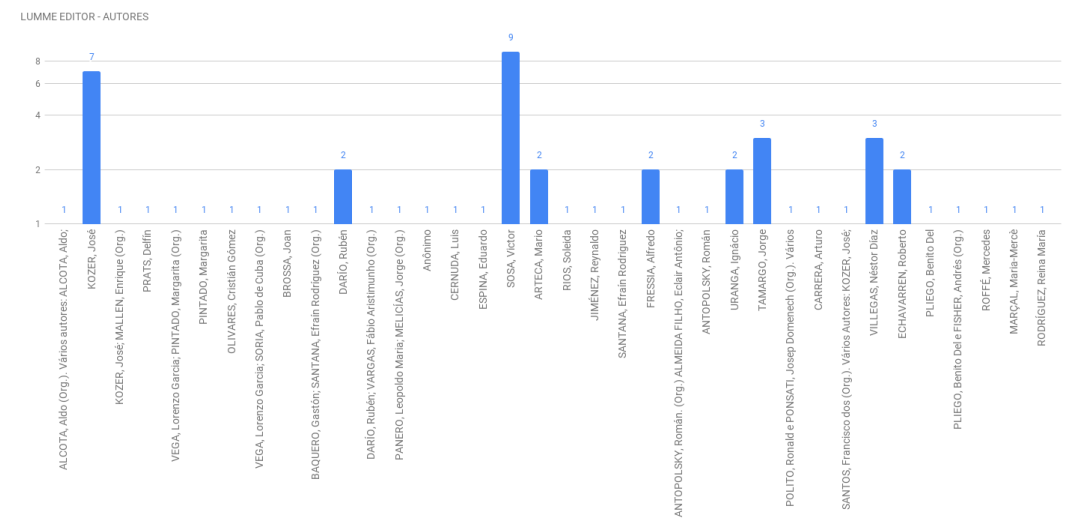

Figura 7 - Lumme Editor - Autores

El gráfico anterior demuestra que, además de poetas canónicos como Rubén Darío existe un interés en la publicación de autores exiliados, como los propios Kozer y Sosa, además de los cubanos Néstor Díaz de Villegas y Jorge Tamargo, entre otros. Si bien Lumme Editor ha publicado diversos libros de Kozer, sin duda el que más se destaca es Nulla dies sine linea (2015), volumen que reúne toda la poesía del cubano, de 1966 a 2015 (10.250 poemas).

A modo de conclusión de estos apuntes, verificamos el lugar secundario que ocupa la poesía hispanoamericana en territorio brasileño respecto a las traducciones del inglés; observamos que los criterios para la selección apuntan a un interés por registrar la presencia de autores vivos y a movimientos 
específicos como el surrealismo, el neobarroco y, en menor medida, a la poesía conceptual. Por último, también constatamos que son las editoriales independientes las que más difunden la poesía hispanoamericana en Brasil.

\section{REFERENCIAS BIBLIOGRÁFICAS}

Milton, John. "Translated Poetry in Brazil 1965-2004". In: Revista Brasileira de Linguistica Aplicada, v. 4, n. 1, 2004, p. 173-193.

Perlongher, Néstor. Caribe Transplatino. São Paulo: Iluminuras, 1991.

Daniel, Claudio. "Revistas definem o panorama literário". In: Musa Rara. 6 de mayo de 2013. Disponible en: https://www.musarara.com.br/revistas-definemo-panorama-literario. Acceso 20 ene. 21.

Demarchi, Ademir. "Babel poética: a poesia na era Lula". In: Espantalhos. Florianópolis: Nave Poética, 2017, p. 125-145.

Kozer, José. "Neobarroco: um convergente padráo na poesia latino-americana”. Trad. de Vima Teixeira. In: Zunái, 2004. Disponible en: http://www.revistazunai. com/ensaios/jose_kozer_neobarroco.htm. Acceso 20 ene. 21.

Mubarack, Chayenne O. "A tradução e publicaçáo de poesia hispano-americana no Brasil: José Kozer", 2016 (artigo inédito).

Pereira, Caroline Costa. "O mundo editorial de Floriano Martins". In: Agulha Revista de Cultura. 6 de junio de 2016. Disponible en: http://arcagulharevistadecultura. blogspot.com/2016/06/caroline-costa-pereira-o-mundo.html. Acceso 20 ene. 21

sem autor. "Brazilian Poetry Journals of the 21st Century". In: Sibila. Revista de poesia e crítica literária. Ano 20, 18 de abril de 2009. Disponible en: https:// sibila.com.br/poetry-essays/novos-olhos-sobre-a-poesia-brazilian-poetryjournals-of-the-21st-century/2419 Acceso 20 ene. 21.

sem autor. "Dossiê Traduçáo". In: Revista Caracol, n. 1, 2010. 